\title{
De La Salle au Canada
}

\section{Daniel Gosselin}

Volume 35, 1968

URI : https://id.erudit.org/iderudit/1007310ar

DOI : https://doi.org/10.7202/1007310ar

Aller au sommaire du numéro

Éditeur(s)

Les Éditions Historia Ecclesiæ Catholicæ Canadensis Inc.

ISSN

0318-6172 (imprimé)

1927-7067 (numérique)

Découvrir la revue

Citer cet article

Gosselin, D. (1968). De La Salle au Canada. Sessions d'étude - Société canadienne d'histoire de l'Église catholique, 35, 99-103. https://doi.org/10.7202/1007310ar

Tous droits réservés @ Les Éditions Historia Ecclesiæ Catholicæ Canadensis Inc., 1968
Ce document est protégé par la loi sur le droit d'auteur. L'utilisation des services d'Érudit (y compris la reproduction) est assujettie à sa politique d'utilisation que vous pouvez consulter en ligne.

https://apropos.erudit.org/fr/usagers/politique-dutilisation/ 


\section{De La Salle au Canada}

Il importe de replacer un homme, un saint, dans son époque, son lignage ancestral, son milieu familial, social, ainsi l'on a plus de chance de saisir dans le concret le vrai sens de sa vie humaine.

Souvent, les hagiographes ont présenté les saints à la manière des monuments élevés à leur mémoire; monument : un bloc de marbre ou de ciment posé sur le sol. Et sur ce socle on élève la statue. Le saint se détache artificiellement de la terre de toute l'épaisseur du support.

Les générations actuelles admirent dans les héros d'aujourd'hui, y compris les saints, leur enracinement humain. Tout ce qui regarde leur hérédité, leur milieu, l'aspect sociologique et caractérologique intéresse beaucoup le chercheur moderne.

Cette mentalité n'est pas neuve. Depuis toujours l'homme demande des dieux "qui marchent devant". Ce besoin profond de l'homme le Fils de Dieu le savait si bien qu'il lui apporte comme réponse sa propre incarnation.

Déjà Léon XIII répondant au cardinal Manning disait: « On a tellement abusé de la manière d'écrire l'histoire, en omettant les choses désagréables. Si les historiens du siècle dernier avait écrit l'Évangile, aujourd'hui nous ne saurions absolument rien du triple reniement de Pierre et de la trahison de Judas, etc. »

Les pages qui vont suivre ont pour objectif de montrer les rapports de saint Jean-Baptiste de La Salle avec le Nouveau-Monde, avec le Canada, en particulier. Ainsi, quand on parlera du Patron des Maîtres, l'on sera plus attentif à l'un des nôtres, de notre race, de notre famille préoccupé des mêmes soucis et travaillant avec nos ancêtres à trouver des solutions satisfaisantes aux problèmes posés par les conditions sociales du temps.

Au contraire de l'angélisme dont se parent certains écrits, de La Salle incarne la réalité la plus prosaïque. Montmorand le présente " doué d'un bon sens que ne trouble aucune exaltation maladive, aucune imagination désordonnée, et auquel s'ajoute la plus rare puissance de pénétration ».

Aîné d'une famille de dix enfants, il vint au monde le 30 avril 1651. Trois membres de sa famille meurent en bas âge. Des sept 
autres, trois se dirigent vers les Ordres: Jean-Baptiste, Jacques-Joseph, Louis. Rose-Marie se fera religieuse. Marie, Pierre et Jean-Rémy se destinent au mariage. A 21 ans il est le tuteur de cette famille.

Dans sa ville natale de Reims où saint Rémi baptisa Clovis; où trente rois reçurent l'onction royale, où sainte Jeanne d'Arc conduisit Charles VII, Jean-Baptiste vivra une quarantaine d'années de sa vie. Le roi Louis XIV règne d'une façon très brillante durant toute cette époque.

C'était de 1651 à 1691. Des grands noms emplissent l'histoire de la France, ne citons que : Colbert, Turenne, Condé, Vauban, Molière, Racine, Corneille, La Fontaine, Pascal, Bossuet, Fénelon. Sans parler des fastes de l'histoire religieuse.

Au Canada, durant ce temps, c'est le défilé des Maisonneuve, Frontenac, LeMoyne, Jolliet, Montigny de Laval, Marguerite Bourgeois, Jeanne Le Ber, des Martyrs Canadiens et cette foule d'explorateurs et de colons, dont l'" histoire est une épopée des plus brillants exploits ».

De La Salle n'est pas parent avec le Cavelier de La Salle, seigneur de Cataracoui, fondateur de Lachine et découvreur du Mississippi. Quand, au Canada, l'on évoque le nom de La Salle, souvent leurs œuvres s'imbriquent et l'on finit par ne plus savoir à qui attribuer l'origine d'une appellation. L'on aura ainsi: Ville-La-Salle, Hôtel La Salle, tabac La Salle, taxi La Salle, etc. Complétant le tableau, l'on trouvera des noms de rue, d'institutions, collèges et académies qui se réfèrent au saint. Un relevé authentique couvrant le Canada et les États-Unis serait intéressant et montrerait aussi le culte que l'histoire a voué à ces pionniers contemporains et compatriotes.

L'essai qui va suivre montre saint $\mathrm{De}$ La Salle mêlé aux fondations sulpiciennes de Montréal. Des compagnons de Séminaire, des professeurs se dévouent pour la colonie naissante et les Relations des Jésuites ainsi que les lettres personnelles occupent des heures de loisirs des étudiants de Saint-Sulpice de Paris.

Deux de ses parents, les pères Jacques Marquette et Jacques Frémin, jésuites, figurent au nombre des missionnaires et explorateurs restés célèbres au Canada.

Pierre Rémy, curé de Lachine, représente un compagnon de Séminaire, pendant que l'un des élèves de l'école normale de Saint JeanBaptiste de La Salle, Antoine Forget, s'adonne à l'éducation des garçons, témoin de l'influence du saint en terre canadienne. En brossant un tableau rapide des premières fondations en Amérique l'on voit, dès 1718, quatre Frères devant s'embarquer pour le Canada. Cette démarche tourne à rien et l'on relève des essais de fondations, s'approchant de 
plus en plus de Montréal : Martinique, Louisiane, Guyane pour aboutir à l'établissement de 1837, à Ville-Marie. Pendant ce temps les Frères Charron entretiennent des rapports continuels avec leurs collègues français et se font un devoir d'utiliser leurs procédés pédagogiques.

Partout c'est le même souffle qui anime ces fondateurs, foi profonde, zèle et dévouement inlassable, centré sur l'enfant, Sa Majesté l'Enfant.

Les compatriotes de 1968 se demandent parfois si l'enfant n'est pas condamné à mourir de froid et d'isolement dans les savantes structures scolaires élaborées à coup de recherches sérieuses empruntées aux cerveaux humains ou électroniques?

L'on n'y échappera pas. Rien ne remplacera l'amour positif, le dévouement, la disponibilité et la gratuité. Les jeunes d'aujourd'hui sont toujours sensibles à ces valeurs profondes. "Seul l'amour est digne de foi.» Nous avons sans doute beaucoup de professeurs: combien avons-nous de MAÎTRES véritables?

En parcourant des pages nombreuses, durant une quarantaine d'années, je suis resté étonné et édifié de voir tant de générosité sous des climats souvent très durs et en des conditions où ne s'étalaient ni luxueuses maisons ni bien-être exagéré. Cependant l'on a trouvé des volontaires pour ces tâches et souvent recrutés chez des sujets n'atteignant pas la vingtaine.

Que l'on ait foi à nos jeunes d'aujourd'hui, qu'on les invite à des missions difficiles et qui en valent la peine et l'on aura encore des engagés, des mordus qui sauront rendre le son de sacrifice, presque à jet continu, à la suite de nos bâtisseurs de pays.

Dans un savant article sur le CEGEP actuel, un connaisseur était obligé d'admettre que l'on fait encore de la ségrégation entre le riche et le pauvre, le bien doué et le retardé. De Le Salle avait été le promoteur de l'éducation populaire. Au service du peuple il avait instauré l'enseignement simultané et l'étude en français ou dans leur langue respective. Évolution? Ce révolutionnaire, comme on l'appelait alors, faisait tout accepter par les grands quand il s'agissait de nosseigneurs les pauvres.

Ainsi dans un humble secteur de Montréal il y a quelques années l'on avait un tableau d'honneur d'étudiants où figuraient cinq évêques actuels. Avant De La Salle, cette promotion sociale était impensable. Seuls les riches pouvaient se payer des études! Qu'il me soit permis de dire toute l'admiration que je continue d'entretenir pour:

a) Jean-Baptiste de La Salle, prêtre, chanoine de Reims, docteur en théologie qui saura vaincre son installation et les douceurs de la 
vie dans une famille riche et noble pour se vouer corps et biens à l'éducation des pauvres;

b) Antoine Forget, laïc, qui à l'imitation des grands modèles qui l'entourent quitte tout, vient au Canada, s'y dévoue durant quinze ans et continue d'y épuiser sa vie gratuitement, seulement pour sa nourriture et son logement. Il meurt en France, procureur du Séminaire d'Angers.

c) La pléiade des fondateurs qui s'expatrient pour assurer l'éducation chrétienne. Ils ont compris "tout ce que vous faites au plus petit d'entre les miens, c'est à moi que vous le faites 》.

Les essais polyvalents de Jean-Baptiste de La Salle en font un initiateur d'une modernité incomparable. Les options graduées, l'école professionnelle, le pensionnat pour les riches, la formation des cinquante jeunes nobles irlandais qui ont accompagné Jacques II leur roi exilé, la «pension de correction» pour les jeunes libertins, la « pension de force » pour les adultes, les cours d'adultes, le séminaire de maîtres d'écoles, la fondation d'un institut, etc., tout cela sous le souffle de l'Esprit et "quand même il ne faudrait vivre que d'aumône et de pain seulement ", selon son vœu héroïque.

Fernand Buisson saluait De La Salle "ce précurseur intelligent qui consacra 40 années du dévouement, le plus patient. le plus inépuisable à l'œuvre obscure dont il était à peu près le seul en France à deviner l'importance et la grandeur ».

Et le sectaire Gustave Lebon renchérissait en affirmant à son tour: « La première chose à faire pour rivaliser avec les Frères serait d'étudier leurs méthodes. Nous sommes libres d'avoir des opinions religieuses fort différentes des leurs, mais nous devons tâcher d'acquérir assez d'indépendance d'esprit pour reconnaître leur supériorité, si manifestement écrasante... Leur sauvage expulsion (de France, 1904) doit être considérée comme un désastre national. »

Mon vœu serait d'obtenir plus de recherches sur les origines de l'Église pour tout ce qui regarde ce "registre immortel \.

L'on écrit parfois et l'on proclame sur les ondes et à la télévision que le monde commence avec nous, du siècle de lumière. En étudiant l'histoire on serait obligé de reconnaître tout le sérieux de cet avertissement de l'Écriture: "Rien de nouveau sous le soleil » et ce serait une belle invitation à un peu plus de modestie.

En finale, je voudrais saluer tous les éducateurs des catégories diverses depuis le papa et la maman qui assurent un bon lancement, jusqu'aux professeurs des différents niveaux. Celui qui façonne la jeunesse détient en mains l'avenir du monde. 
Hommage particulier aux confrères lasalliens et à ceux des congrégations-sœurs qui, animés du même souffle missionnaire œuvrent sous tous les continents. A côté des 18,000 lasalliens ils sont 52,000 des autres instituts. Leur présence et leur dévouement redisent au monde "le signe que le Seigneur est venu : les pauvres sont évangélisés ».

Tout ne s'est pas arrêté avec la mort de Jean-Baptiste en 1719. Par ses écrits à l'usage des maîtres et des élèves il a continué de faire école.

Ses disciples aussi ont compris qu'il n'avait pas voulu être une borne, mais un phare. A leur tour ils sont allés porter le flambeau de la foi et de la civilisation chrétienne, s'adaptant aux pays qui les accueillaient, parlant la langue du pays et adoptant les coutumes.

Les membres de la Société Historique de l'Ouest du Québec m'avaient demandé de trouver des références à notre région. Cela n'est pas difficile puisque les Marquette et Frémyn sont venus ici. La Place du Portage et l'Asticou (ou Chaudière) étaient bien connues de ces explorateurs et missionnaires.

Je retiens cette invitation muette qu'ils pourraient nous adresser: \& allez aux faits »!

C'est la suggestion que faisait également Pie XII à des collégiens de Rome: "Ne faites pas comme des enfants mal éduqués. Ouvrez le livre de l'Histoire avec beaucoup de respect. Ne détruisez pas les pages du passé. Ce serait de l'infantilisme. Au contraire, en vous référant à l'exemple des devanciers servez-vous-en comme tremplin pour un dépassement. Ecrivez bien votre page, à votre tour. » Je conclus: il faudra que le monde soit meilleur parce que nous aurons vécu, bien vécu!

Frère Daniel Gosselin, é.c., Coordonnateur de la catéchèse aux diocèses d'Ottawa et de Hull. 